\title{
Pain as the Presenting Symptom in Renal Tuberculosis
}

\author{
E. J. G. MILROY, K. OWEN
}

British Medical fournal, 1971, 1, 642-643

\section{Summary}

Four cases of renal tuberculosis presented with severe renal pain. A search should be made for tuberculous bacilluria in patients with renal pain of no obvious aetiology.

\section{Introduction}

Both the overall death rate and the notification rate of cases of tuberculosis are falling, but the rates for non-respiratory tuberculosis remain unchanged (Fig. 1). The overall decline in incidence is accounted for by the fall in the number of pulmonary cases, and though it is reasonable to expect a similar fall in genitourinary cases after a time lag of 8-10 years (Obrant, 1955), the present incidence of new cases of genitourinary tuberculosis and the difficulty in diagnosis continue to give rise to concern.

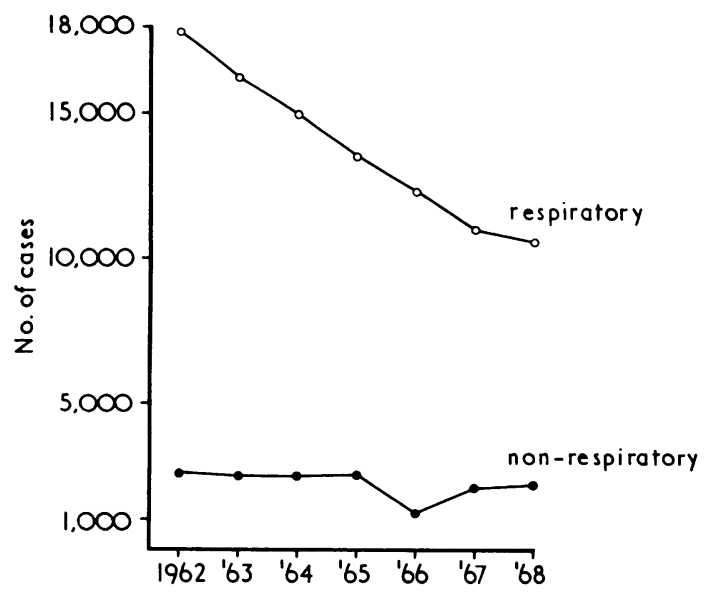

FIG. 1-Notification rates for tuberculosis. Department of Health and Social Security.

Renal tuberculosis frequently presents with a variety of mild symptoms, often with cystitis, sometimes associated with haematuria. Renal pain when it occurs is usually mild and of an aching character. Renal tuberculosis is not often considered in the differential diagnosis of severe renal pain. We have recently seen four patients whose main presenting symptom was severe renal pain in whom diagnosis of tuberculosis was made after some delay and after more obvious causes for the pains had been excluded.

\section{Case 1}

A woman medical practitioner aged 26 had a severe attack of right loin pain six weeks before presenting. This was followed by further attacks of similar pain, on one occasion with macroscopic

St. Mary's Hospital, London W.2

E. J. G. MILROY, F.R.C.S., Senior Registrar

K. OWEN, M.S., F.R.C.S., Consultant Urologist haematuria, but with no other urinary symptoms. Her urine was sterile on routine culture and contained no white cells. An intravenous pyelogram was thought to be normal but there was some suspicion over the left upper calix, the side opposite to the renal \& pain. Early morning urine specimens were then taken and these grew Mycobacterium tuberculosis. Standard triple drug therapy was started three months after she first presented, and in the subsequent six months there have been no further attacks of renal pain.

\section{Case 2}

A post office clerk aged 36 presented with recurrent attacks of severe colicky left groin pain radiating to the groin. Each attack $\stackrel{+}{N}$ lasted one to two hours and they had been occurring every few weeks for the previous five months. He had no associated dysuria, frequency, or haematuria. His urine was sterile on routine culture and contained only occasional white cells. The original pyelogram showed a distorted left lower calix with some calcification, and his chest $x$-ray film, originally reported as normal, in retrospect showed old calcified foci in the right upper zone. The diagnosis of tuberculosis was confirmed on culture from early morning urine specimens, and he was started on standard triple drug therapy one year after first presenting. Two months after treatment started a stricture developed at the lower end of the left ureter, which had to be reimplanted into the bladder. He has had no further attacks of loin pain in the eight years since his operation.

\section{Case 3}

A nurse aged 25, whose father had died of pulmonary tuberculosis, presented with a two-year history of recurrent attacks of colicky right-sided loin pain, originally with no other urinary symptoms but more recently associated with dysuria and frequency. Though her original urine cultures were sterile with only occasional white cells, she was diagnosed as having recurrent urinary infections and pyelonephritis, with some "psychological overlay." Her original intravenous and retrograde pyelograms were normal. With continuing symptoms, early morning urine specimens were taken. Löwenstein cultures were repeatedly negative, but two years after she had first presented tuberculosis was confirmed on guinea-pig inoculation, and treatment was started with standard triple drug therapy. She has had no further attacks of pain in the subsequent five years.

\section{Case 4}

An Italian business man aged 50, gave an 18-month history of recurrent attacks of severe right-sided loin pains, sometimes colicky, sometimes a severe continuous ache. During the first attack of colic he noticed some haematuria but had seen none since and had noticed no dysuria or frequency. His urine was sterile on routine culture and contained no cells. Repeated cultures for tubercle bacilli were negative. An intravenous pyelogram showed an apparently normal right kidney, but on the left there appeared to be a large cavity or very dilated upper calix which filled slowly (Fig. 2). A retrograde pyelogram failed to fill the left upper pole calix. Selective renal arteriography showed a normal vascular pattern on the right side with a normal nephrogram; on the left side the upper pole was ill defined and appeared to be supplied by an accessory artery.

The left kidney was explored and a greatly expanded and almost completely destroyed upper pole was found. Upper pole partial nephrectomy was performed. Culture of the excised kidney failed to grow tubercle bacilli. Macroscopic examination (Dr. R. C. B. 


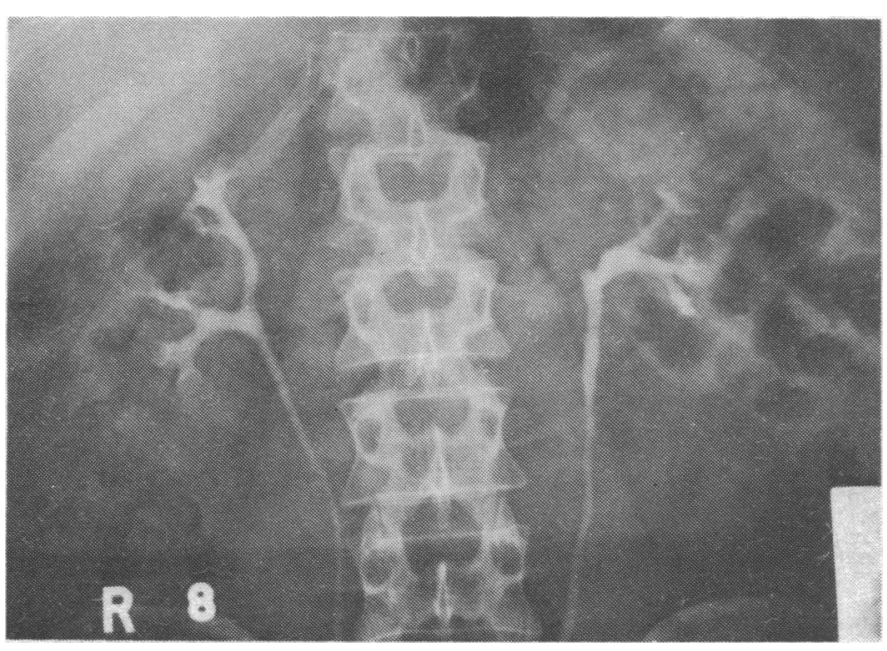

FIG. 2-Case 4. Intravenous pyelogram-early film showing delay in filling left upper calix. Apparently normal right kidney.

Pugh) showed a grossly dilated upper pole calix containing chalky material; microscopically the dilated calix was lined by tuberculous granulation tissue which in many areas had undergone organization and hyalinization. Though no acid-fast bacilli were seen the appearances were entirely consistent with the diagnosis of partially healed renal tuberculosis. He was started on standard triple drug therapy four months after first presenting, and in the subsequent 18 months has had no further attacks of pain in either kidney.

\section{Discussion}

Severe renal pain is usually said to be uncommon as a presenting symptom of renal tuberculosis, normally being associated in the more advanced cases with haematuria, the passage of tuberculous debris, or ureteric strictures (Wells, 1943; Freshman, 1962).

All our four cases presented with severe unilateral renal pain not associated with other urinary symptoms, though two had haematuria at some stage in the disease. The diagnosis of tuberculosis was not considered when they first presented because of the absence of "typical" symptoms of tuberculous disease and the absence of pyuria in all the patients. The initial pyelograms suggested tuberculosis in two patients but were normal in the other two. Case 4 was of particular interest in that he presented with right renal colic and was found on pyelography to have advanced left-sided renal tuberculosis with an apparently normal right kidney. After starting antituberculous therapy his pain disappeared completely, and was therefore presumably due to tuberculous involvement of the contralateral kidney. The renal pains have not returned in any of the four patients after starting standard drug therapy.

Tuberculosis is known as a great imitator. Wechsler et al. (1960) recommended a high index of suspicion regarding patients with vague renal colic, finding an incidence of $8 \%$ of new cases presenting with renal pain. In some larger series 4.6\% (Borthwick, 1956) and 9\% (Obrant 1955) of cases presented with pain as a main symptom. Nicholls (1953) went further and said that a parade of victims of the disease would present as an assortment of healthy-looking men and women.

Our purpose in reporting these cases is to encourage the search for genitourinary tuberculosis in all patients presenting with renal pain in whom a definite alternative diagnosis cannot be made. Emergency pyelography has reduced the number of cases presenting with renal pain being clinically diagnosed as "gravel" in which no radiological abnormality is found. Repeated urine culture for tuberculosis in these cases may further reduce their number.

We are grateful to Dr. R. C. B. Pugh for the histological report on Case 4.

\section{References}

Borthwick, W. M. (1956). Tubercle, 37, 120.

Freshman, E. (1962). Proceedings of the Royal Society of Medicine, 55, 968 Nicholls, M. F. (1953). In Modern Trends in Urology, ed. E. W. Riches, p. 357. London, Butterworth.

Obrant, O. (1955). Acta Chirurgica Scandinavica, 110, 3.

Wechsler, H., Westfall, M., and Lattimer, J. K. (1960). Fournal of Urology $83,801$.

Wells, C. (1943). Postgraduate Medical Fournal, 19, 270.

\title{
Value of Re-examining X-ray Films of Outpatients Attending Accident Services
}

\author{
C. S. B. GALASKO, P. R. W. MONAHAN
}

\section{British Medical fournal, 1971, 1, 643-644}

\section{Summary}

$X$-ray films of 4,665 patients who had attended an accident service as outpatients were reviewed by the clinicians and reported on by the radiologist the next day.

In 20 cases the radiologist did not comment on bony injuries which had been diagnosed and treated by the accident service senior house officer. The radiologist, however, detected bony injuries which had been missed by the accident service senior house officer in 26 cases. In 10 cases injuries

Accident Service, Radcliffe Infirmary, Oxford

C. S. B. GALASKO, ch.M., F.R.c.s., Senior Registrar

P. R. W. MONAHAN, M.B., F.R.C.S., Senior Registrar (Current address : Nuffield Orthopaedic Centre, Oxford) which had not been detected at the original examination or by the radiologist were noted when the $x$-ray films were reviewed. On two occasions the fracture was missed at all three examinations.

The daily joint review of $x$-ray films by accident service officers and the radiologist seemed to increase the clinical and radiological acumen of the staff of the accident service.

\section{Introduction}

Most patients attending accident services or casualty departments have relatively minor injuries, which are usually dealt with by the senior house officer on duty. These injuries may occur at times when a radiologist's opinion of the $x$-ray films is not immediately available. The severer injuries are usually seen by a more senior member of the team and, if the patient 